\title{
Synthesis and Aggregation Behavior of a Glycolated Naphthalene Diimide Bithiophene Copolymer for Application in Low-Level n-Doped Organic Thermoelectrics
}

Young-hun Shin, Hartmut Komber, Davide Caiola, Marco Cassinelli, Hengda Sun, Dominik Stegerer, Marcel Schreiter, Kilian Horatz, Franziska Lissel, Xuechen Jiao, Christopher R. McNeill, Simone Cimò, Chiara Bertarelli, Simone Fabiano, Mario Caironi, and Michael Sommer*

Cite This: Macromolecules 2020, 53, 5158-5168

Read Online

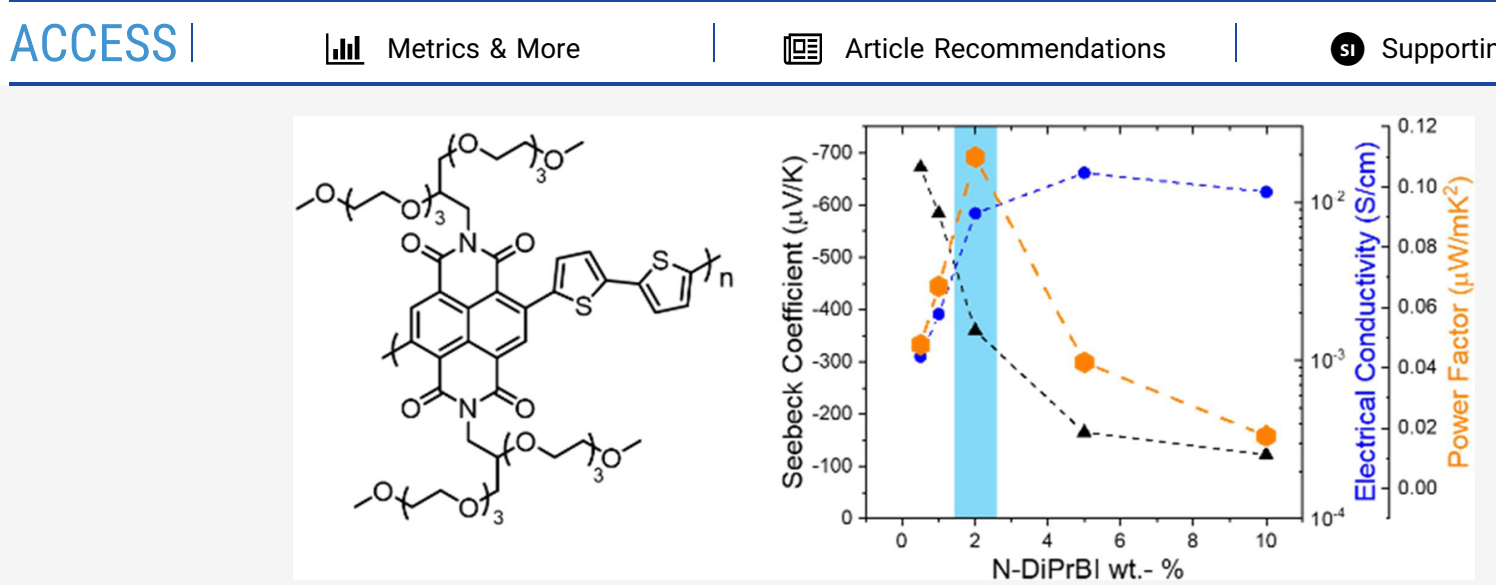

ABSTRACT: The synthesis of a naphthalene diimide bithiophene copolymer P(EO-NDIT2) with branched, base-stable, and purely ether-based side chains is presented. Stille polycondensation leads to high molecular weights that are limited by methyl transfer and eventually T2 homocouplings. While extensive solution aggregation hampers molecular weight determination by conventional methods, NMR spectroscopy allows identification of both T2- (H and methyl) and NDI-related (methyl) end groups, enabling the determination of absolute number average molecular weights larger than $M_{\mathrm{n}, \mathrm{NMR}} \sim 100 \mathrm{~kg} / \mathrm{mol}$. Solvent- and temperature-dependent aggregation in solution is investigated by NMR and UV-vis spectroscopy. These results are used for solution doping of $\mathrm{P}(\mathrm{EO}-$ NDIT2) with N-benzimidazole-based n-dopants. Spin coating from heated chlorobenzene solutions and using 4-(2,3-dihydro-1,3dimethyl-1H-benzoimidazol-2-yl)- $N, N$-diisopropylaniline (N-DiPrBI) as the dopant leads to homogeneous films with highest conductivities up to $10^{-2} \mathrm{~S} / \mathrm{cm}$. Generally, N-DiPrBI concentrations as low as $\sim 5 \mathrm{wt} \%$ are sufficient to increase conductivity by orders of magnitude. Strikingly, maximum power factors up to $0.11 \mu \mathrm{W} / \mathrm{mK}^{2}$, although limited by conductivity, are achieved for the highest molar mass sample at a low dopant concentration of 2 wt \% N-DiPrBI only.

\section{INTRODUCTION}

Recent years have witnessed tremendous progress in the development of semiconducting conjugated polymers with engineered side chains. ${ }^{1}$ In particular, polar side chains with ether, amine, or ionic functionalities endow conjugated materials with a diversified property profile and thus enable further applications. Examples are bioelectronics, organic electronics, and organic thermoelectrics. ${ }^{2-8}$ Here, the polar side chains give rise to numerous advantages over their purely aliphatic counterparts such as processability from polar, environmentally benign, and also orthogonal solvents, ${ }^{9-11}$ good interaction with aqueous electrolytes, ${ }^{2,4,12}$ increased dielectric constant, ${ }^{6}$ reduced $\pi$ - $\pi$ stacking distance, ${ }^{3}$ improved miscibility with dopant molecules, ${ }^{5}$ and electrical conductivity of organic electrochemical transistors (OECTs). ${ }^{4}$ Kroon et al. ${ }^{13}$ published a polythiophene with oligo ethylene oxide (EO) side chains that showed high electrical conductivity up to 100 $\mathrm{S} / \mathrm{cm}^{2}$ after p-type doping. Chen et al. ${ }^{6}$ reported diketopyrrolopyrrole-based conjugated polymers with a polar branched side chain that exhibited $>5 \%$ power conversion efficiency when used as the donor material in organic solar cells processed from spin-coating. Recently, research on naphthalene diimide bithiophene copolymers (PNDIT2), triggered by pioneering works of Guo and Watson and Facchetti et al., ${ }^{14,15}$

Received: March 20, 2020

Revised: June 12, 2020

Published: July 1, 2020 
with EO-based side chains was disclosed. ${ }^{2,4,5,16-18}$ Giovanitti et al. demonstrated water-stable, n-type OECTs from PNDIT2 with both polar side chains at the NDI and T2 building blocks. ${ }^{2,4}$ Kiefer et al. investigated the thermoelectric performance of the same material and found an increased doping efficiency with the n-dopant 4-(2,3-dihydro-1,3-dimethyl-1Hbenzoimidazol-2-yl)-N,N-dimethylaniline (N-DMBI). ${ }^{5}$ Further, NDI copolymers with linear EO side chains at mostly the NDI unit were reported by Liu et al. and Wang et al., who reported high electrical conductivities and power factors up to $1.8 \mathrm{~S} / \mathrm{cm}$ and $4.5 \mu \mathrm{W} / \mathrm{mK}^{2}{ }^{17-19}$ An interesting amphiphilic side chain structure was used for the synthesis of an NDI copolymer by Kim et al., who disclosed increased electron mobilities above $1 \mathrm{~cm}^{2} / \mathrm{Vs} .{ }^{16}$ All of these works made use of linear EO side chains (Scheme 1a), which do not endow NDI

Scheme 1. Overview of (a) Literature-Known Polar, Ethylene Oxide Based Side Chains Used for NDI Copolymers $^{4,16,18}$ and (b) Side Chains Made in This Work

a) NDI monomers with linear EO side chains

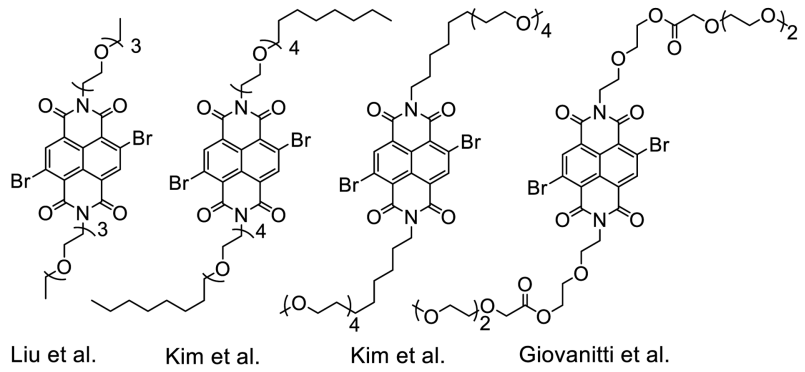

b) NDI monomers with branched EO side chains (this work):
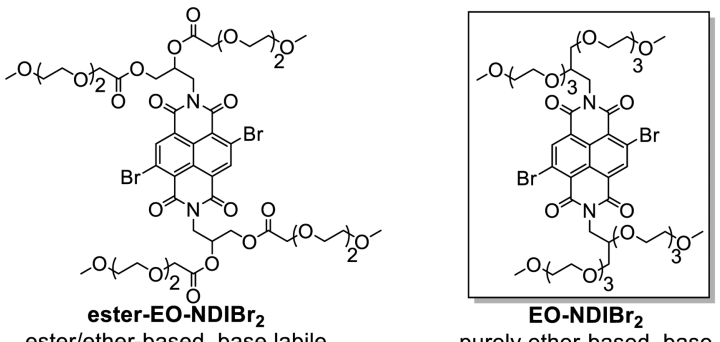

EO-NDIBr

purely ether-based, base stable

copolymers with sufficiently high solubility and thus limit molecular weight (MW). In case the NDI comonomer carries additional side chains, increased MW is possible though. ${ }^{4,18}$ However, while it is surprising that materials with oligomeric character show much improved performance, ${ }^{17}$ clarification of a possible MW dependence is a prime question when dealing with polymeric materials. Moreover, from a synthetic design point of view and to broaden comonomer range, NDI copolymers with polar side chains and significantly increased solubility are desirable.

Here, we report the synthesis of PNDIT2 with branched, purely EO-based side chains (Scheme $1 \mathrm{~b}$ ) that allow the preparation of highly soluble, high-MW EO-PNDIT2. Compared to mixed ester/ether functionality side chains, the branched EO side chains are stable under chromatography conditions and further allow the use of polycondensation techniques involving a base. Detailed NMR spectroscopy including model compounds and end group analysis enabled detection of defect structures and calculation of absolute number average molecular weights not possible by other methods, and MALDI-ToF mass spectrometry (MS) further confirmed both structure and end group analysis. Optical, thermal, and electrical properties of $\mathrm{P}(\mathrm{EO}-\mathrm{NDIT} 2)$ were further investigated in detail, revealing unusual aggregation behavior in solution, backbone aggregation with increased segregated stacking mode in thin films, and an increasing doping efficacy compared to PNDIT2 with aliphatic side chains. $^{20,21}$

\section{RESULTS AND DISCUSSION}

Inspired by the drastically different properties of conjugated polymers with polar side chains, ${ }^{4,13,17}$ we were interested in high-MW PNDIT2 with EO side chains for applications in which conjugated backbones are jointly present with ions. ${ }^{22-24}$ Using an adapted protocol from Giovanitti et al., ${ }^{4}$ we first attempted a one-pot procedure for the synthesis of branched ester-EO-NDIBr 2 (Schemes $1 \mathrm{~b}$ and $2 \mathrm{a}$ ) with mixed ester and ether functionalities. While this monomer can be made in low yield in one step, the ester group is unstable and is cleaved under standard column chromatography and direct arylation polycondensation conditions. Therefore, we used a purely ether-based, branched side chain, which should equip the resulting copolymer with good stability and high solubility in organic solvents. ${ }^{25}$ Starting from ( \pm )-3-amino-1,2-propanediol, we prepared 2,3-bis(methoxy(triethyleneoxide))propan-1amine (EO-amine) in three steps ${ }^{26-29}$ and an overall yield of $82 \%$ (Scheme 2b). Subsequently, EO-amine was reacted with 2,6-dibromonaphthalene dianhydride $\left(\mathrm{NDABr}_{2}\right)$ to obtain the monomer $\mathrm{EO}-\mathrm{NDIBr}_{2}$ in $11 \%$ yield. The relatively low yield of NDI monomers (Scheme 1a, 13-27\%) and EO-NDIBr 2 was mainly caused by the presence of regioisomers of $\mathrm{NDABr}_{2}$, which were only removed after imidization, which significantly complicated purification. First attempts to copolymerize EO$\mathrm{NDIBr}_{2}$ with 2,2'-bithiophene using direct arylation polymerization $^{30}$ gave low yields and oligomeric samples. Stille coupling was employed instead, giving $\mathrm{P}(\mathrm{EO}-\mathrm{NDIT} 2)$ with high MW and yield (Scheme 2c). The resulting molecular weights were mostly influenced by the solvent and monomer purity. A summary of entries and reaction conditions is compiled in Table 1. Purification by Soxhlet extraction using ethyl acetate, acetone, and chloroform gave chloroform fractions in $52-90 \%$ yields (Table 1).

The conditions for Stille polycondensation were optimized regarding the solvent. Anisole gave low MWs but, interestingly, the highest yields. Chlorobenzene yielded higher MWs, and DMF yielded the highest ones. The most influential parameter affecting MW was not the solvent but monomer purity. Therefore, a slight excess of 1.16 equiv. was used compared to the stannylated bithiophene comonomer (Table 1), which was shown to improve molar mass development. ${ }^{31-33}$

MW determination was approached first using size exclusion chromatography (SEC) in chloroform. However, unusually high values on the order of $10^{3}-10^{5}$ and $10^{5}-10^{6} \mathrm{~kg} / \mathrm{mol}$ for $M_{\mathrm{n}}$ and $M_{\mathrm{w}}$, respectively, were obtained with PS calibration (Figure S1). Extreme aggregation of conjugated polymers, especially of those carrying EO side chains, ${ }^{13}$ is not unknown but is usually caused by backbone interactions. Moreover, aggregates observed in SEC are often accompanied by molecularly dissolved chains, which was not observed here. Notably, UV-vis spectroscopy of P(EO-PNDIT2) in chloroform suggested nonaggregated backbones (Figure S2), while SEC in chloroform and at the same concentration did not 
Scheme 2. Synthesis of (a) Ester-EO-NDIBr 2 , (b) EO-NDIBr, , (c) P(EO-NDIT2), and (d) Model Compounds

a)<smiles></smiles><smiles>COCCOCCOCC(=O)O</smiles>

$\underset{140^{\circ} \mathrm{C}, 16 \mathrm{~h}}{\stackrel{o-x y l e n e}{\longrightarrow}}$

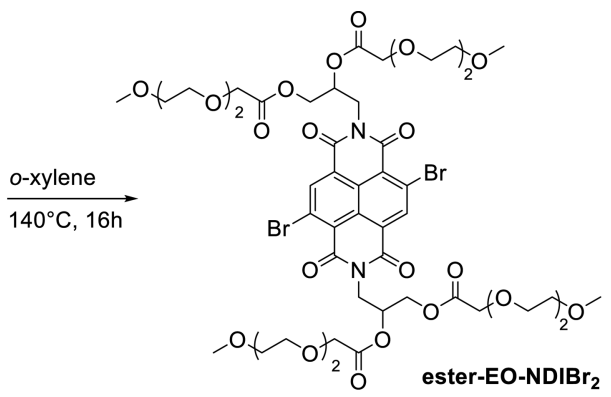

b)
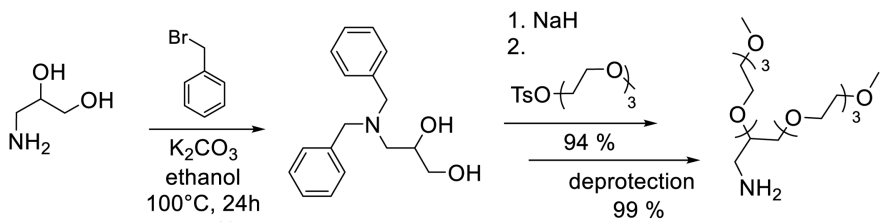

EO-amine
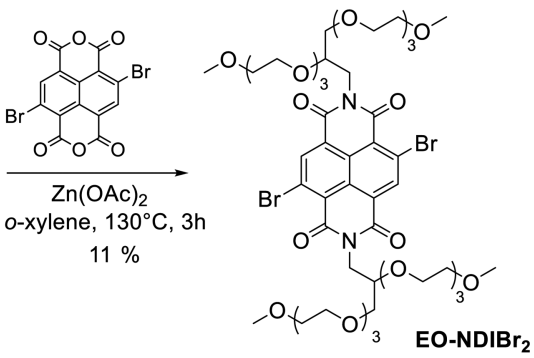

c)

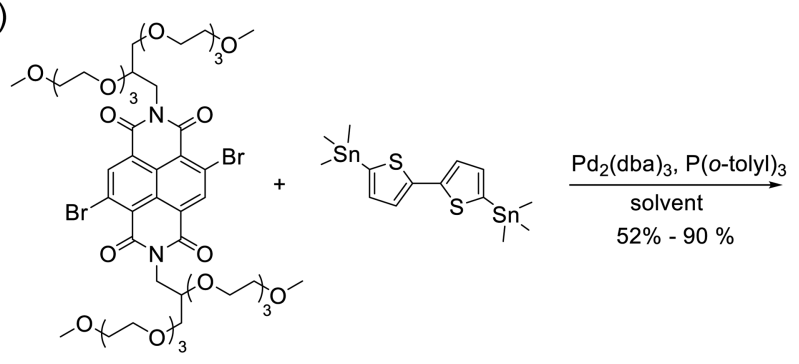

EO-NDIBr

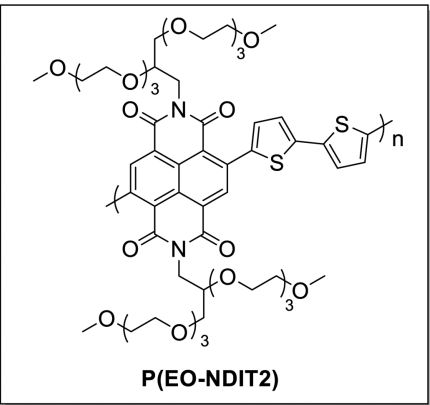

d)

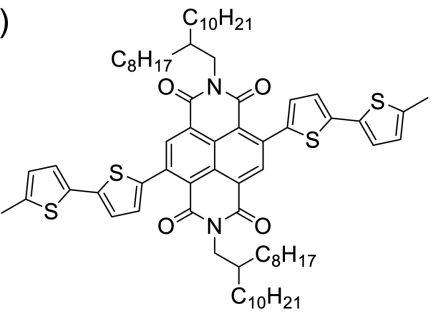

Me-T2-NDI-T2-Me

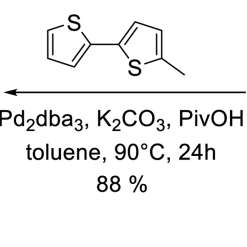

$88 \%$

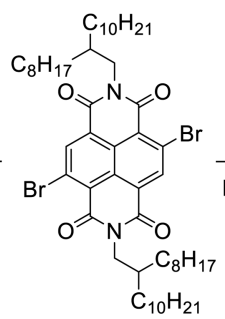

$\mathrm{NDIBr}_{2}$

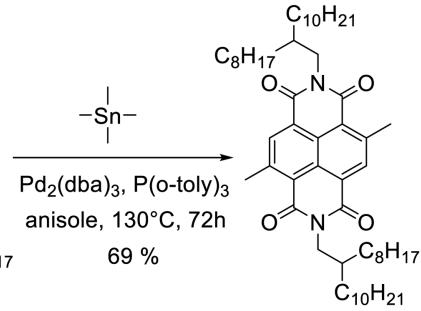

Me-NDI-Me

Table 1. Summary of Reaction Conditions and Molecular Weight of P(EO-NDIT2 $)^{a}$

\begin{tabular}{|c|c|c|c|c|c|c|c|}
\hline entry & ratio EO-NDI/T2 & solvent $(\text { conc })^{b}$ & $T\left({ }^{\circ} \mathrm{C}\right)$ & $\mathrm{DP}_{\mathrm{n}, \mathrm{NMR}}{ }^{c}$ & $M_{\mathrm{n}, \mathrm{NMR}}(\mathrm{kg} / \mathrm{mol})^{c}$ & $M_{\mathrm{n}, \mathrm{SEC}} / M_{\mathrm{w}, \mathrm{SEC}}(\mathrm{kg} / \mathrm{mol})^{d}$ & yield $(\%)^{e}$ \\
\hline 1 & 1.1 & $\mathrm{AN}(0.05)$ & 130 & 8.5 & 11 & & 90 \\
\hline 2 & 1 & $\mathrm{AN}(0.05)$ & 130 & 24 & 27 & & 84 \\
\hline 3 & 1.16 & $\mathrm{AN}(0.05)$ & 130 & 25 & 29 & & 84 \\
\hline 4 & 1.16 & $\operatorname{DMF}(0.02)$ & 85 & 25 & 30 & & 71 \\
\hline 5 & 1.1 & DMF (0.02) & 85 & 33 & 38 & & 81 \\
\hline 6 & 1.1 & $\mathrm{CB}(0.05)$ & 130 & 35 & 42 & $1.2 \times 10^{3} / 6.0 \times 10^{5}$ & 76 \\
\hline 7 & 1.1 & DMF (0.02) & 85 & 35 & 42 & $3.8 \times 10^{3} / 2.77 \times 10^{5}$ & 69 \\
\hline 8 & 1.1 & $\mathrm{CB}(0.05)$ & 130 & 62 & 73 & $1.21 \times 10^{4} / 9.14 \times 10^{5}$ & 52 \\
\hline 9 & 1.1 & DMF (0.02) & 85 & 100 & 116 & $4.32 \times 10^{5} / 3.2 \times 10^{6}$ & 77 \\
\hline
\end{tabular}

${ }^{a} \mathrm{CB}$, DMF, and AN are chlorobenzene, dimethylformamide, and anisole, respectively. $\operatorname{Pd}_{2}(\mathrm{dba})_{3}(1 \mathrm{~mol} \%)$ and $\mathrm{P}(o \text {-tolyl })_{3}(4 \mathrm{~mol} \%)$ were used for all entries; reaction time was $48 \mathrm{~h}$ except for entry $8(24 \mathrm{~h}) .{ }^{b}$ Concentration of stannylated bithiophene in mol/L. ${ }^{c}$ From NMR end group analysis; estimated error $\pm 10 \% .{ }^{d}$ From $\mathrm{SEC}$ in $\mathrm{CHCl}_{3}$ at room temperature. ${ }^{e}$ Isolated yield of the $\mathrm{CHCl}_{3}$ fraction after Soxhlet extraction with ethyl acetate, acetone, and $\mathrm{CHCl}_{3}$. 
show any molecularly dissolved chains. On the one hand, these results suggest a rather unusual, possibly micellar-type aggregation with segregated EO side chains, and on the other hand, these call for alternative MW determinations. Therefore, NMR end group analysis was applied to determine the degree of polymerization $\mathrm{DP}_{\mathrm{n}, \mathrm{NMR}}$ and number average molecular weight $M_{\mathrm{n}, \mathrm{NMR}}$ (Table 1 ).

Different from PNDIT2 with common, branched 2octyldodecyl (OD) side chains, ${ }^{34}{ }^{1} \mathrm{H}$ NMR spectra of $\mathrm{P}$ (EOPNDIT2) in $\mathrm{CDCl}_{3}$ gave sufficient resolution at room temperature. Nevertheless, the measurements were carried out in $\mathrm{C}_{2} \mathrm{D}_{2} \mathrm{Cl}_{4}$ at $120{ }^{\circ} \mathrm{C}$ to refer to previous studies on structural defects of PNDIT2, such as end groups or homocouplings. In order to calculate the degree of polymerization from main chain and end group signal intensities, all end groups must be known. Unlike PNDIT2 made via DAP, ${ }^{30}$ end groups of PNDIT2 made via Stille polycondensation are not always well characterized by NMR spectroscopy. ${ }^{35}$ In order to assign observed end group signals (Figure 1a,c), two OD-substituted model compounds were synthesized mimicking methyl end groups at the T2 and NDI chain ends (Scheme
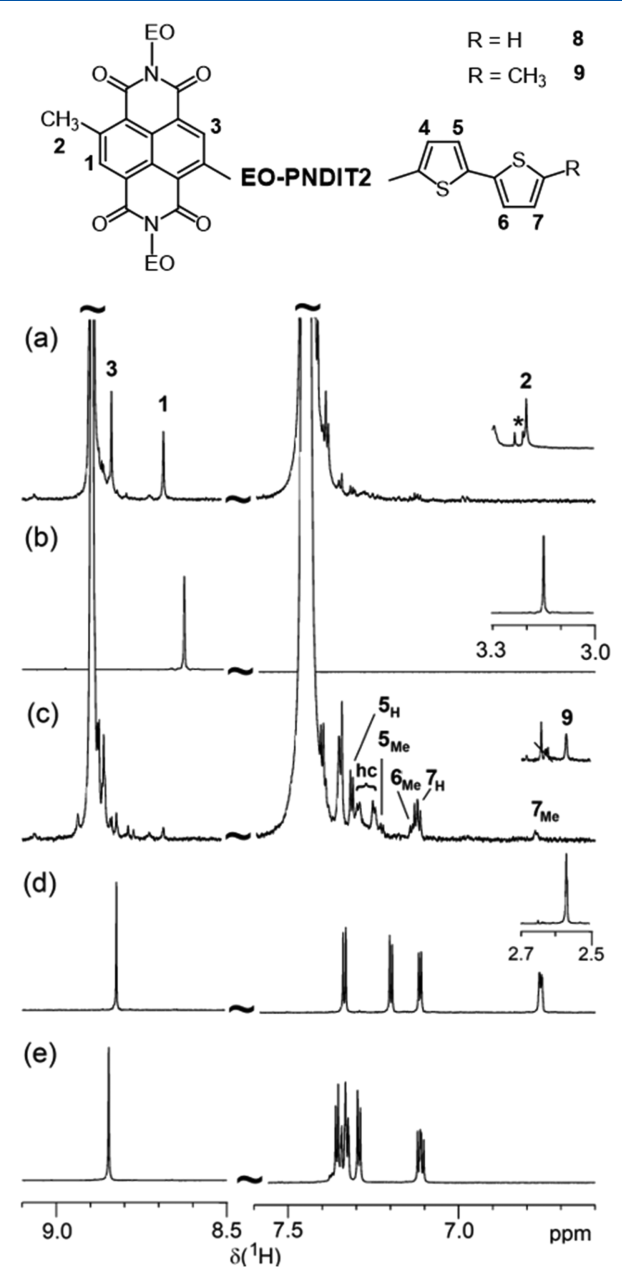

Figure 1. ${ }^{1} \mathrm{H}$ NMR spectra (regions) of (a) Me-NDI-terminated $\mathrm{P}$ (EO-PNDIT2) (entry 6), (b) Me-NDI-Me, (c) Me-T2- and H-T2terminated EO-PNDIT2 (entry 2), (d) Me-T2-NDI-T2-Me, and (e) $\mathrm{H}$-T2-NDI-T2-H (all in $\mathrm{C}_{2} \mathrm{D}_{2} \mathrm{Cl}_{4}$ at $120^{\circ} \mathrm{C}$ ). The model compounds are 2-octyldodecyl substituted at the imide nitrogen. Asterisk marks a ${ }^{13} \mathrm{C}$ satellite signal of the $\mathrm{OCH}_{3}$ group, and hc designates signals from T2-T2 homocouplings. 2d). The model compound Me-T2-NDI-T2-Me was synthesized in two steps. First, Me-T2 was synthesized by Suzuki coupling of 2-bromo-5-methylthiophene and 2-thienylboronic acid pinacol ester to get a colorless liquid in $94 \%$ yield. Subsequently, OD-NDIBr${ }_{2}$ was reacted with an excess of MeT2 under direct arylation conditions to get Me-T2-NDI-T2$\mathrm{Me}$ as a bluish powder in $88 \%$ yield (see the Supporting Information). The second model compound Me-NDI-Me was obtained by Stille coupling between OD-NDIBr 2 and $\mathrm{SnMe}_{4}$ (Scheme 2d, Supporting Information).

The comparison of the NMR spectra of these two model compounds (Figure 1b,d and Figures S6 and S7) and the polymer spectra clearly proved methyl termination both at the NDI (3.20 ppm) and T2 chain ends $(2.57 \mathrm{ppm})$. The methylNDI end group signals could also be assigned by ROESY measurements on a polymer sample (Figure S8). While methyl end groups of conjugated polymers made by Stille polycondensation are not uncommon, ${ }^{36}$ the chemical shifts for the methyl groups found with PNDIT2 were unknown to our knowledge. The third end group that has to be considered for the calculation of $\mathrm{DP}_{\mathrm{n}, \mathrm{NMR}}$ is the common $-\mathrm{T} 2-\mathrm{H}$ end group (Figure 1e). The dominant presence of methyl termination at both comonomers can be explained by undesired transmetallation of a methyl group instead of a $\mathrm{T} 2$ group from the stannylated bithiophene comonomer. The relatively intense Me-NDI end groups result from the slight excess of EO$\mathrm{NDIBr}_{2}$ monomer used, which accelerates molar mass formation at early stages of polycondensation. ${ }^{31}$ From these end group intensities, absolute number average molecular weights $M_{\mathrm{n}, \mathrm{NMR}}$ were calculated (Table 1 ). While these values can be regarded as an upper limit, it is clear that they are much smaller than values from SEC, suggesting NMR spectroscopy to be the method of choice here.

Notably, for entry 2, two slightly broadened doublets at 7.29 and $7.25 \mathrm{ppm}$ were observed pointing to a backbone defect (Figure 1c). Comparison with the spectrum of PNDIT4 reported in a previous study ${ }^{30}$ reveals these signals to arise from T2-T2 homocoupling (Figure S9), which is a known side reaction in Stille reactions involving stannylated T2 building blocks. $^{37}$

Further information on end groups was obtained from MALDI-ToF MS analysis (Figure 2). In general, the detection of PNDIT2 with MALDI-ToF MS is not trivial, probably due to strong aggregation, and signals are often confined to lowmolecular-weight fractions. Prior reports show that the stepwise replacement of alkyl by EO side chains improves the detectability of PNDIT2 and also enhances the quality of the collected spectra. A potential explanation for this is the ability of polyether units to form oxonium ions. For P(EONDIT2), the branched EO side chains lead to well-defined signals with high intensities, allowing not only retrieval of structural confirmation but also information about end groups. Based on the MALDI-ToF MS results, the calculated average molar mass of the repeating unit is $1162.1 \mathrm{Da} \pm 0.4$, consistent with the theoretical molar mass of $1161.3 \mathrm{Da}$. The positive mode MALDI-ToF mass spectrum (Figure 2) contains four signal series, two major and two minor ones, of which three can be clearly assigned to different end group combinations: (1) a doubly methyl-NDI terminated chain (red), (2) a methyl-NDI and T2-H terminated chain (green), and (3) a doubly $\mathrm{T} 2-\mathrm{H}$ terminated chain (orange). Consistent with the NMR analyses, a dominant presence of methyl termination is observed, as well as an excess of NDI termini. Signals are 


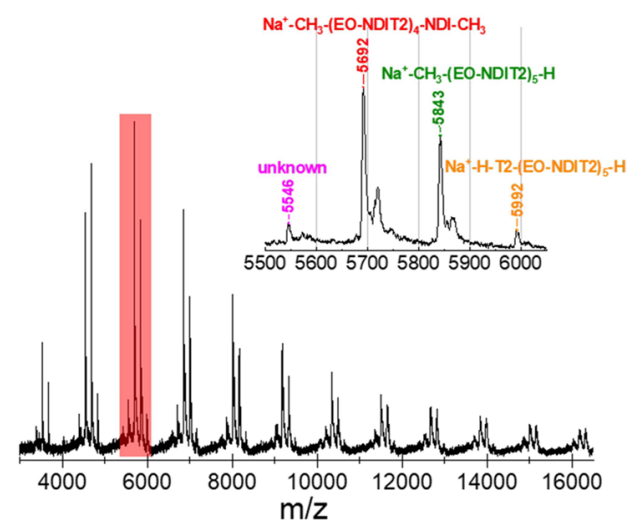

Figure 2. Reflectron positive mode MALDI-ToF mass spectra of P(EO-NDIT2) (entry 9) with trans-2-[3-(4-tert-butylphenyl)-2methyl-2-propenylidene]malononitrile (DCTB) (20:1) under addition of $1 \mathrm{vol} \%$ sodium trifluoroacetate (NaTFA). The inset shows the four dominant signal series. Note that, for the green series, the two end groups can also be reversed.

detected until $\sim 30 \mathrm{~kg} / \mathrm{mol}$. Still, it was not possible to calculate reliable molecular weight and dispersity $(Ð)$ values due to the discrimination against higher-molecular-weight species. Further details can be found in the Supporting Information (Figures S10-S12).

Having investigated the molecular structure, end groups, and homocouplings as side reactions, ${ }^{1} \mathrm{H}$ NMR spectroscopy was further used to probe solvent-dependent aggregation at $30{ }^{\circ} \mathrm{C}$ on a qualitative basis (Figure 3). Significant broadening of NDI
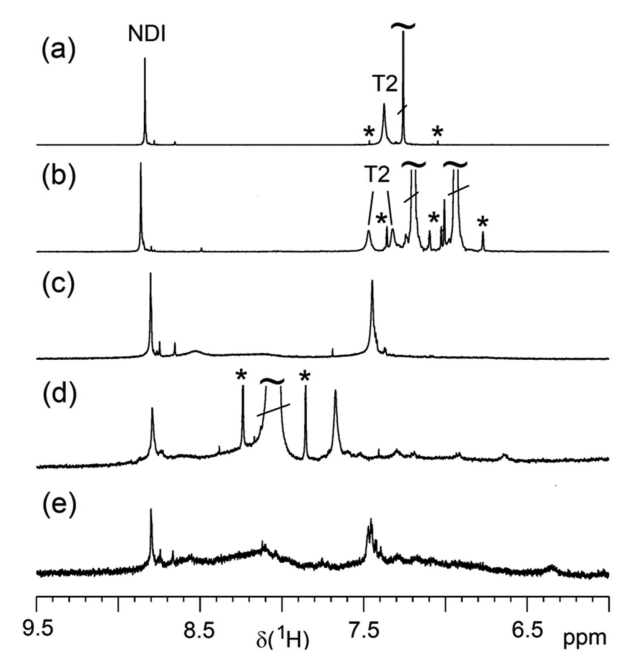

Figure 3. Aggregation of $\mathrm{P}(\mathrm{EO}-\mathrm{PNDIT} 2)$ (entry 3 ) in solution at 30 ${ }^{\circ} \mathrm{C}$ probed by ${ }^{1} \mathrm{H}$ NMR spectroscopy. (a) $\mathrm{CDCl}_{3}$, (b) o-DCB- $d_{4}$, (c) dioxane- $d_{8}$, (d) DMF- $d_{7}$, and (e) THF- $d_{4}(4.2 \mathrm{mg} / \mathrm{mL})$. Asterisk marks ${ }^{13} \mathrm{C}$ satellites of the solvent signal. Note that the expected two signals of the T2 unit show up only in $o$-DCB- $d_{4}$, whereas in all other solvents, both protons have the same chemical shift.

and thiophene signals accompanied by shielding/deshielding effects indicates $\pi-\pi$ stacking of these backbone units within aggregates. $^{34}$ The spectra further show that aggregation as a result of $\pi-\pi$ stacking is strongly suppressed in chloroform $\left(\mathrm{CDCl}_{3}\right)$ and ortho-dichlorobenzene (o-DCB) (Figure 3a,b). In dioxane (DX), both narrow signals of molecularly dissolved chains and broad signals of aggregates appear (Figure 3c). In contrast, both dimethylformamide (DMF) and tetrahydrofuran
(THF) show extensive aggregation (Figure 3d,e), with THF being the worst solvent. A general feature observed for poor solvents is that signals of chain termini appear narrower than backbone signals pointing to a higher solvation at such defects in the aggregates. Dissolution should progress from such defects. Thus, solvent quality at $30{ }^{\circ} \mathrm{C}$ decreases with the order $\mathrm{CHCl}_{3}, o-\mathrm{DCB}, \mathrm{DX}, \mathrm{DMF}$, and THF. The order of decreasing solvent quality was also investigated by UV-vis spectroscopy where a new band at $\sim 750 \mathrm{~nm}$ continuously develops with decreasing solvent quality $\left(\mathrm{CHCl}_{3}, o-\mathrm{DCB}, \mathrm{DMF}\right.$, and $\left.\mathrm{DX}\right)$ (Figure S2). The low solubility of P(EO-PNDIT2) in THF did not allow UV-vis spectra to be obtained even at low concentration and elevated temperature.

Increasing temperature resulted in further dissolution of aggregates, with different temperatures being required for the different solvents to fully dissolve aggregates (Figures 4 and 5

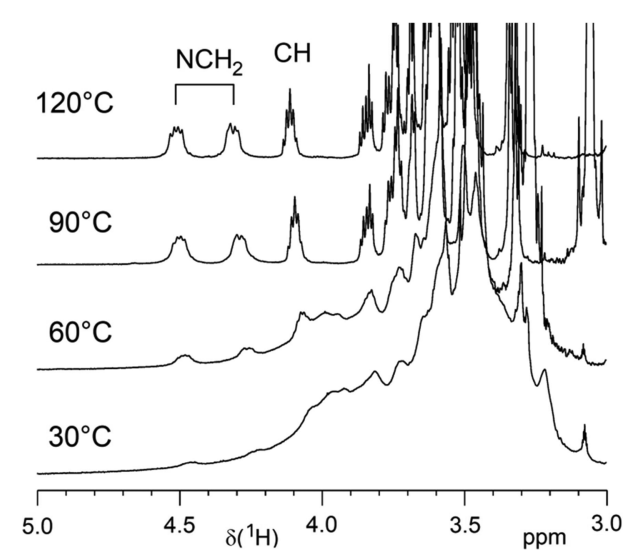

Figure 4. Temperature-dependent aggregation of $\mathrm{P}(\mathrm{EO}-\mathrm{PNDIT2})$ (entry 3 ) in DMF- $d_{7}$ probed by ${ }^{1} \mathrm{H}$ NMR spectroscopy (see also Figure S14 for spectra with $10 \mathrm{~K}$ steps). The region of the EO side chain signals is shown.

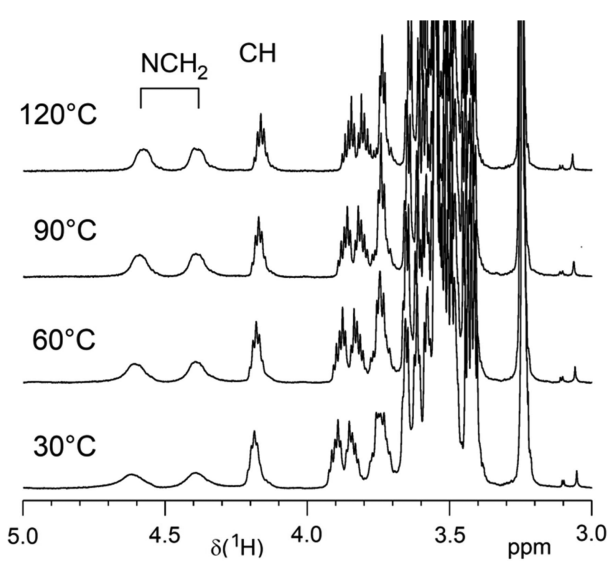

Figure 5. Temperature-dependent aggregation of $\mathrm{P}(\mathrm{EO}-\mathrm{PNDIT2})$ (entry 3 ) in $o$-DCB- $d_{4}$ probed by ${ }^{1} \mathrm{H}$ NMR spectroscopy. The region of the EO side chain signals is shown.

and Figures S13 and S14). The temperature dependence was investigated for the two high-boiling-point solvents $o$-DCB and $\mathrm{DMF}$, in which a rather low and high degree of aggregation are present at $30{ }^{\circ} \mathrm{C}$ (Figures S1 and S2). Figure 4 shows temperature-dependent ${ }^{1} \mathrm{H}$ NMR spectra in DMF- $d_{7}$ between 30 and $120{ }^{\circ} \mathrm{C}$. Here, the region of side chain protons is depicted (aromatic protons' region in Figure S13a). Because the $\mathrm{NCH}_{2}$ group next to the NDI core is affected by 
aggregation, its signal also appears broadened. Molecularly dissolved chains were present at temperatures between 120 and $90{ }^{\circ} \mathrm{C}$, where all signals were well resolved. Upon cooling, signals became again broader and less resolved. At $30{ }^{\circ} \mathrm{C}$, these signals become very broad and new signals appear reflecting new chemical environments in the aggregates (Figure S14). Thus, DMF is a good solvent at high temperature but a moderate one at RT. This explains both the relatively high degree of aggregation at RT seen by UV-vis spectroscopy, as well as the fact that high molecular weights can be synthesized at $85{ }^{\circ} \mathrm{C}$ in this solvent. Figure 5 shows the same plot recorded in $o$-DCB- $d_{4}$ (aromatic protons' region in Figure S13b). The onset of aggregation is indicated by slight signal broadening at $\sim 60{ }^{\circ} \mathrm{C}$. Clearly, aggregation at $30{ }^{\circ} \mathrm{C}$ is much less pronounced than in DMF. This is in accordance with UV-vis spectroscopy, where the shoulder at $750 \mathrm{~nm}$ is less developed than in DMF.

To further investigate chain aggregation in solution at different temperatures and its reversibility, temperaturedependent UV-vis spectroscopy was carried out in $o$-DCB and CB (Figure 6). These two solvents were selected because of their relevance for solution processing as well as the fact that solvent quality is different (Figure S2). To this end, one heating and cooling cycle from 20 to $100{ }^{\circ} \mathrm{C}$ was used, and absorption was measured stepwise every $20 \mathrm{~K}$. In $o$-DCB, the intensity of the band at $750 \mathrm{~nm}$ reduced from 20 to $40{ }^{\circ} \mathrm{C}$ and was entirely absent at $60{ }^{\circ} \mathrm{C}$ (Figure 6a). Interestingly, this band was not restored upon cooling with the absorption spectrum remaining unchanged even after prolonged storage of the solution at $20{ }^{\circ} \mathrm{C}$ for 2 weeks (Figure $6 \mathrm{~b}$ ).

The same experiment was performed in $\mathrm{CB}$ (Figure 6c,d). Qualitatively, heating a solution of P(EO-NDIT2) (entry 9) in $\mathrm{CB}$ also leads to dissolution of aggregates, albeit higher temperatures were necessary compared to $o$-DCB. In $\mathrm{CB}$, aggregates only fully dissolved between 60 and $80{ }^{\circ} \mathrm{C}$. Another difference was that aggregates re-formed upon cooling until RT. Interestingly, storage of CB solutions at RT led to further development of the band at $750 \mathrm{~nm}$ during a few weeks.

Thin films were further characterized by cyclic voltammetry (CV) and UV-vis spectroscopy, which showed significant differences from PNDIT2 with aliphatic side chains. P(EONDIT2) exhibits a slightly lowered LUMO energy level of $-3.88 \mathrm{eV}$ compared to PNDIT2 $(-3.75 \mathrm{eV})$. The typical twoelectron reduction $\mathrm{CV}$ curve of PNDIT2 is not observed with $\mathrm{P}$ (EO-NDIT2), for which the second reduction is drastically reduced in intensity (Figure S15). UV-vis thin-film spectra further indicate a more intense band at $\sim 750 \mathrm{~nm}$ (Figures S2 and S16) and generally different shapes from PNDIT2. Different polymorphs are possible according to Brinkmann et al., ${ }^{38}$ but a distinct stacking mode could not be determined.

To gain further understanding on $\mathrm{P}(\mathrm{EO}-\mathrm{NDIT} 2)$ thin-film morphology, grazing-incidence wide-angle $\mathrm{X}$-ray scattering (GIWAXS) was employed to study films spin cast from $\mathrm{CB}$ and $o$-DCB. Both films exhibit characteristic lamellar stacking, backbone stacking, and $\pi-\pi$ stacking peaks as seen from the two-dimensional GIWAXS patterns shown in Figure S17. Reduced one-dimensional line profiles are presented in Figure 7, taken along the horizontal (in-plane) and vertical scattering (out-of-plane) directions. Along the in-plane direction, the lamellar stacking $(h 00)$ and backbone stacking (00l) peaks are obviously stronger for films processed from CB than for films processed from $o$-DCB. The same trend holds true for the $\pi$ - $\pi$ stacking (010) peak seen in the out-of-plane direction. This suggests that $\mathrm{CB}$ is more capable than $o-\mathrm{DCB}$ of facilitating the
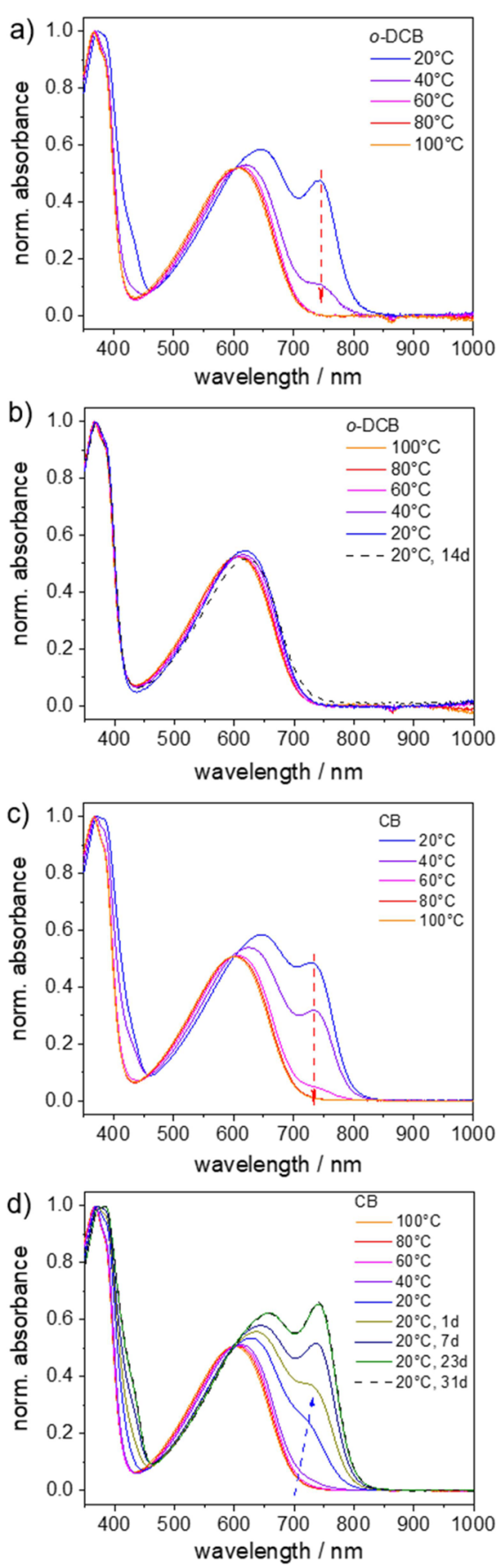

Figure 6. UV-vis spectra of $\mathrm{P}(\mathrm{EO}-\mathrm{NDIT} 2)$ (entry 9) by heating from 20 to $100{ }^{\circ} \mathrm{C}$ and cooling from 100 to $20^{\circ} \mathrm{C}$ in $(\mathrm{a}, \mathrm{b}) o$-DCB and $(\mathrm{c}, \mathrm{d}) \mathrm{CB}$.

formation of ordered aggregates, which is consistent with what is observed from temperature-dependent UV-vis measurements. Further details including lattice spacing and crystallite size (approximated by the coherence length) have been extracted via multiple peak fitting analysis and are summarized as shown in Table 2. From these data, processing from $o$-DCB is found to slightly decrease the lattice spacing of lamellar, backbone, and $\pi-\pi$ stacking peaks. Intriguingly, the influence of different solvents on crystallite size is more considerable. The usage of CB largely enhances the crystallite size along all three crystallographic directions. More specifically, the crystallite sizes increase from 9.8 to $17.4 \mathrm{~nm}$, from 12.6 to $17.9 \mathrm{~nm}$, and 


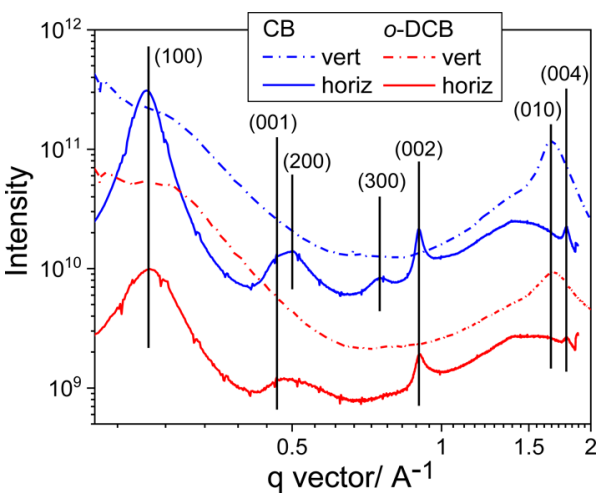

Figure 7. One-dimensional GIWAXS scattering profiles of films prepared from $\mathrm{CB}$ (blue) and $o$-DCB (red) corresponding to vertical (dash-dotted) and horizontal (solid) directions.

Table 2. Key Microstructural Parameters Extracted from the GIWAXS Data

$\begin{array}{clcc} & & \text { P(EO- } & \text { P(EO-NDIT2) } \\ & & \text { NDIT2) CB } & \text {-DCB } \\ \text { in-plane lamellar } & Q\left(\AA^{-1}\right) & 0.255 & 0.259 \\ & d(\AA) & 24.63 & 24.25 \\ & \text { FWHM }\left(\AA^{-1}\right) & 0.036 & 0.064 \\ & \text { coherence length } & 174.44 & 98.13 \\ \text { in-plane backbone } & Q(\AA) & & \\ (001) & Q\left(\AA^{-1}\right) & 0.464 & 0.474 \\ & d(\AA) & 13.53 & 13.25 \\ & \text { FWHM }\left(\AA^{-1}\right) & 0.035 & 0.050 \\ & \text { coherence length } & 179.43 & 125.6 \\ \text { out-of-plane } \pi-\pi & (\AA) & & 1.682 \\ (010) & Q\left(\AA^{-1}\right) & 1.679 & 3.73 \\ & d(\AA) & 3.74 & 0.282 \\ & \text { FWHM }\left(\AA^{-1}\right) & 0.187 & 22.27 \\ & \text { coherence length } & 33.58 & \\ & (\AA) & & \end{array}$

from 2.2 to $3.4 \mathrm{~nm}$ along the lamellar, backbone, and $\pi$ - $\pi$ stacking directions, respectively.

Thermal analysis further indicates a lower thermal stability as well as a lower melting point of $\mathrm{P}$ (EO-NDIT2) than PNDIT2 and that the EO side chains are able to crystallize as well (Figures S18 and S19). For these reasons, solution doping was employed, and thermal annealing protocols were not applied to thin films.

To investigate electrical conductivity upon doping, $\mathrm{P}(\mathrm{EO}-$ NDIT2) (entry 4) was solution-doped with several $\mathrm{N}$ benzimidazole-based n-dopants for doping ratios of $0-30 \mathrm{wt}$ $\%$ and subsequently processed into films. Among the different $\mathrm{N}$-benzimidazoles used for doping, 4-(2,3-dihydro-1,3-dimethyl-1H-benzoimidazol-2-yl)-N,N-diisopropylaniline (N-DiPrBI) gave the best results at moderate doping levels (Figure S20). ${ }^{39,40}$ Considering the results of aggregation of $\mathrm{P}(\mathrm{EO}-$ NDIT2) in solution, we selected $o-\mathrm{DCB}$ and $\mathrm{CB}$ as solvents for solution doping and processing. Both solvents are good solvents in general, allow for convenient processing, but lead to various degrees of order as discussed. $o$-DCB solutions were heated to $90{ }^{\circ} \mathrm{C}$ to dissolve aggregates, and the dopant was added. In the case of $\mathrm{CB}$, addition of N-DiPrBI and processing of the mixture were done at room temperature where aggregates are present, as well as at $80{ }^{\circ} \mathrm{C}$, where aggregates are dissolved (see Figure 6). Nonheated $o$-DCB solutions were not considered as the corresponding UV-vis spectra were very similar to those of CB.

Both the solvent and the temperature protocol have an effect on conductivity (Figure 8). For all doping protocols,

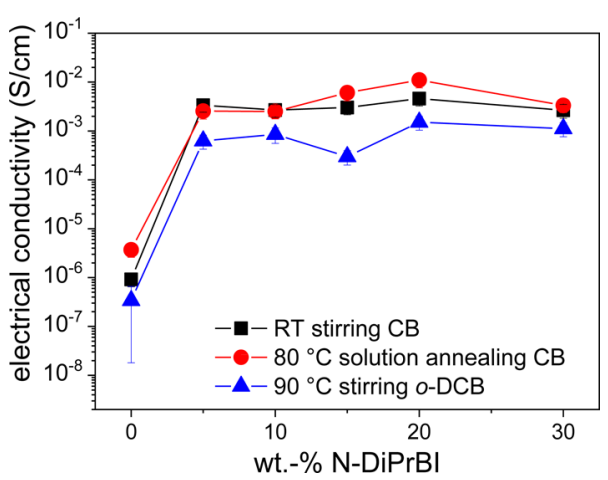

Figure 8. Electrical conductivity of $\mathrm{P}(\mathrm{EO}-\mathrm{NDIT} 2)$ (entry 4) films doped with N-DiPrBI.

conductivity increased over several orders of magnitude to $\sim 10^{-3}$ to $10^{-2} \mathrm{~S} / \mathrm{cm}$ between 0 and 5 wt $\%$ doping. For larger doping levels, only small changes in conductivity were seen. Notably, the highest conductivities were found for $\mathrm{CB}$ solutions annealed at $80{ }^{\circ} \mathrm{C}$, a temperature at which aggregates are dissolved.

These results can be understood qualitatively by correlation with the results from GIWAXS as well as with surface topographies from atomic force microscopy (AFM). Figure 9
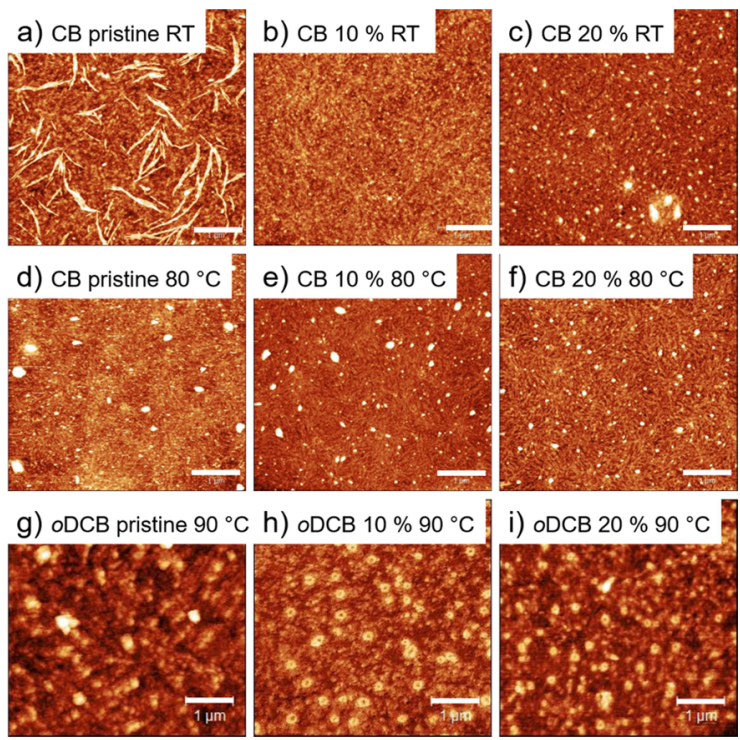

Figure 9. Atomic force microscopy (height images) on thin films of $\mathrm{P}$ (EO-NDIT2) (entry 4) spun from $(\mathrm{a}-\mathrm{c}) \mathrm{CB}$ at room temperature, $(\mathrm{d}-\mathrm{f}) \mathrm{CB}$ heated to $80^{\circ} \mathrm{C}$, and $(\mathrm{g}-\mathrm{i}) o$-DCB heated to $90^{\circ} \mathrm{C}$. $(\mathrm{a}, \mathrm{d}, \mathrm{g})$ Pristine films, (b,e,h) 10 wt \% N-DiPrBI, and $(\mathrm{c}, \mathrm{f}, \mathrm{i}) 20$ wt $\% \mathrm{~N}$ DiPrBI. The scale bar is $1 \mu \mathrm{m}$ for all images.

shows a series of surface images for the three protocols and in pristine and doped states. Films spun from $o$-DCB were rougher and exhibited nodular morphologies (Figure 9g-i), while films from $\mathrm{CB}$ were smoother and displayed fibrillar morphologies (Figure 9a-f). In addition, films spun from $C B$ solutions that were not heated to $80{ }^{\circ} \mathrm{C}$ showed $\mu \mathrm{m}$-sized, large aggregates on top of the film but only in the undoped 
state (Figure 9a). The ability of $\mathrm{P}(\mathrm{EO}-\mathrm{NDIT} 2)$ to crystallize is also seen by DSC experiments, which show both main chain and side chain melting (Figure S19 and Table S3). Meltannealing of $\mathrm{P}$ (EO-NDIT2), however, is accompanied by degradation, possibly by side chain cross linking of the less stable EO side chains (Figure S18). Upon doping with 10 wt \% $\mathrm{N}-\mathrm{DiPrBI}$, films spun from $\mathrm{CB}$ became yet smoother, indicating excellent compatibility of $\mathrm{P}(\mathrm{EO}-\mathrm{NDIT} 2)$ and $\mathrm{N}$ DiPrBI. The elongated aggregates (Figure 9a) were no longer seen, and new aggregates from dopant segregation were absent as well at $10 \mathrm{wt} \%$ doping. Only at $20 \mathrm{wt} \%$ doping, dot-like features can be discerned, which may point to dopant segregation at the surface; however, this high doping fraction is in fact not needed to achieve maximum conductivities for this system. The origin of ring-like features seen on the surface of doped films spun from $o$-DCB is unclear and was not further investigated here as values for conductivities were moderate (Figure 9h,i).

It is important to note that while higher conductivities have been reported for a different NDI copolymer with polar side chains, ${ }^{5}$ the amount of dopant required to reach the maximum conductivity is lower here. We assume that varying molecular weights and further fine tuning of aggregate formation in $\mathrm{CB}$ solution using temperature, time, and concentration are useful strategies to further improve absolute values of electrical conductivity. The differences in electrical conductivities among the three processing protocols were further correlated with results from UV-vis spectroscopy on pristine and doped films (Figure S21). Figure S21a shows film absorption of as-spun, pristine films of $\mathrm{P}$ (EO-NDIT2) (entry 4). Among the three protocols, processing from non-annealed $\mathrm{CB}$ solution leads to the most intense low-energy absorption band with developed bands. This is in accordance with the observation of large, fiber-like aggregates seen on top of films (Figure 9a). With a $10 \%$ dopant, the low-energy band of films spun from CB at 750 $\mathrm{nm}$ decreases, while increases are observed for the bands at 470 and $820 \mathrm{~nm}$, which are clear signatures of radical anion formation (Figure S21b). ${ }^{41,42}$ Films made from $o$-DCB remained almost unchanged upon doping. This suggests that the lower conductivity of $o$-DCB films is not only caused by a different morphology but additionally by a lower doping efficiency. Both trends continue for 20 wt \% doping (Figure S21c).

The Seebeck coefficients $(S)$ of the doped films were measured under optimized conditions and to estimate the power factor $\mathrm{PF}=S^{2} \sigma$ (Figure 10). Doping levels of 0-10 wt $\%$ were considered for economic reasons as most changes in conductivity occurred within this region. Moreover, possible effects of molecular weight on performance were probed. Figure 10a shows data for entry 6 (moderate molecular weight $M_{\mathrm{n}, \mathrm{NMR}}=42 \mathrm{~kg} / \mathrm{mol}$ ), and Figure $10 \mathrm{~b}$ shows data for entry 9 (high molecular weight $M_{\mathrm{n}, \mathrm{NMR}}=116 \mathrm{~kg} / \mathrm{mol}$ ). To minimize the error in measuring $S$, an optimized contact geometry was used consistently for both molecular weights. For the $42 \mathrm{~kg} /$ mol sample, the Seebeck coefficient decreases continuously with increasing dopant concentration, going from $-550 \mu \mathrm{V} / \mathrm{K}$ for pristine films to $-50 \mu \mathrm{V} / \mathrm{K}$ at $10 \%$ doping. The negative sign of $S$ is consistent, with electrons being the majority carrier type. Since $\sigma$ and $S$ feature opposite trends with increasing charge density, PF optimizes at an intermediate dopant concentration, reaching a value of $0.06 \mu \mathrm{V} / \mathrm{K}$ at only the 1 wt \% doping level (Figure 10a). The higher-molar-mass sample with $116 \mathrm{~kg} / \mathrm{mol}$ showed almost the same conductivity
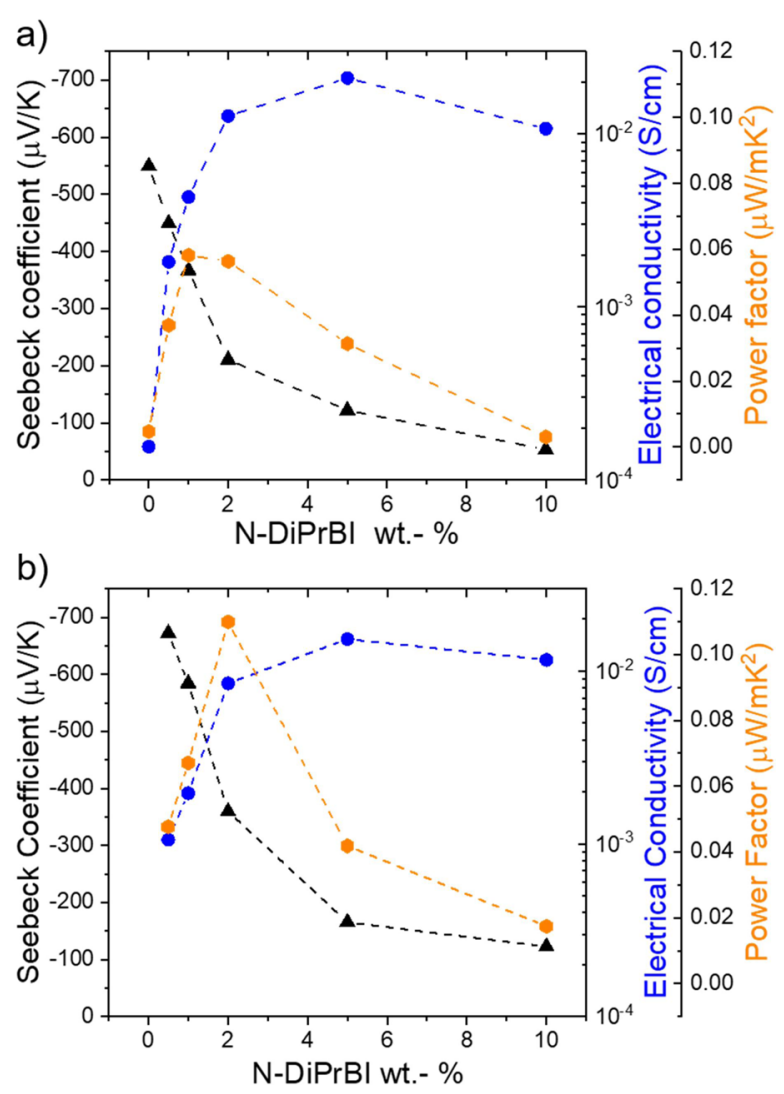

Figure 10. Seebeck coefficient, conductivity, and power factors of $\mathrm{P}($ EO-NDIT2) of different molecular weights. Films were made from solution-annealed CB and doped with N-DiPrBI. (a) entry 6, $42 \mathrm{~kg} /$ $\mathrm{mol}$ and (b) entry $9,116 \mathrm{~kg} / \mathrm{mol}$.

behavior and values but increased Seebeck coefficients, resulting in an almost doubled PF of $0.11 \mu \mathrm{V} / \mathrm{K}$ at 2 wt $\%$ doping (Figure 10b). We observed similar values and trends when P(EO-NDIT2) was exposed to the vapor of tetrakis(dimethylamino)ethylene (TDAE, Figure S22), suggesting intrinsic limitations to maximum conductivities and Seebeck coefficients. Yet, achieving maximum PF values for low doping levels of $1-2 \mathrm{wt} \%$ is highly promising (i) as the amount of dopant needed is minimized and (ii) as miscibility issues are unlikely to play a major role.

\section{CONCLUSIONS}

We have synthesized $\mathrm{P}$ (EO-NDIT2) with branched ethylene oxide (EO) side chains that do not carry ester functionalities. These side chains are base-stable and impart high solubility to PNDIT2. End groups and defects are investigated by detailed NMR and MALDI-ToF MS measurements. Methyl termination during Stille polycondensation is prevalent and is used for the determination of absolute number average molecular weights. Due to its amphiphilic nature, P(EO-NDIT2) exhibits generally very good solubility but also pronounced aggregation in common organic solvents, which may or may not include backbone aggregation depending on the solvent. Chlorobenzene is an especially beneficial solvent to induce and control backbone aggregation, which is helpful to achieve larger crystals, a higher degree of order, and maximum electrical conductivities slightly higher than $\sim 10^{-2} \mathrm{~S} / \mathrm{cm}$ upon doping with the benzimidazole-based dopant N-DiPrBI. Maximum power factors are achieved for low doping levels of $1-2$ wt \% 
only, with higher molecular weight resulting in improved performance. This further emphasizes the important role of polar side chains attached to conjugated polymers for thermoelectric applications. Further investigation of the diverse aggregation phenomena in solution is ongoing and will be the basis for a detailed understanding of the self-assembly behavior of $\mathrm{P}$ (EO-NDIT2), which is critical for further optimization of morphology and the resulting electrical performance.

\section{ASSOCIATED CONTENT}

\section{s) Supporting Information}

The Supporting Information is available free of charge at https://pubs.acs.org/doi/10.1021/acs.macromol.0c00657.

Details of synthesis, measurements, and experimental procedures; additional NMR spectroscopy and MALDIToF MS measurements, SEC, UV-vis spectroscopy, and CV data, 2D GIWAXS patterns, and thermal analysis data (PDF)

\section{AUTHOR INFORMATION}

\section{Corresponding Author}

Michael Sommer - Institut für Chemie, Technische Universität Chemnitz, 09111 Chemnitz, Germany; 이이.org/00000002-2377-5998; Email: michael.sommer@chemie.tuchemnitz.de

\section{Authors}

Young-hun Shin - Institut für Chemie, Technische Universität Chemnitz, 09111 Chemnitz, Germany

Hartmut Komber - Leibniz Institut für Polymerforschung Dresden e. V, 01069 Dresden, Germany; ㅇo orcid.org/00000001-6176-6737

Davide Caiola - Center for Nano Science and Technology @ PoliMi, Istituto Italiano di Tecnologia, Milano 20133, Italy

Marco Cassinelli - Center for Nano Science and Technology@ PoliMi, Istituto Italiano di Tecnologia, Milano 20133, Italy

Hengda Sun - Laboratory of Organic Electronics, Department of Science and Technology, Linköping University, SE-60174 Norrköping, Sweden

Dominik Stegerer - Institut für Chemie, Technische Universität Chemnitz, 09111 Chemnitz, Germany

Marcel Schreiter - Institut für Chemie, Technische Universität Chemnitz, 09111 Chemnitz, Germany

Kilian Horatz - Leibniz Institut für Polymerforschung Dresden e. V, 01069 Dresden, Germany

Franziska Lissel - Leibniz Institut für Polymerforschung Dresden e. V, 01069 Dresden, Germany; Technische Universität Dresden, 01062 Dresden, Germany; 이이이.org/0000-00030254-4565

Xuechen Jiao - Department of Materials Science and Engineering, Monash University, Clayton, Victoria 3800, Australia; o orcid.org/0000-0001-7387-0275

Christopher R. McNeill - Department of Materials Science and Engineering, Monash University, Clayton, Victoria 3800, Australia; (1) orcid.org/0000-0001-5221-878X

Simone Cimò - Center for Nano Science and Technology @ PoliMi, Istituto Italiano di Tecnologia, Milano 20133, Italy; Dipartimento di Chimica, Materiali e Ingegneria Chimica "Giulio Natta", Politecnico di Milano, 20133 Milano, Italy

Chiara Bertarelli - Center for Nano Science and Technology @ PoliMi, Istituto Italiano di Tecnologia, Milano 20133, Italy;
Dipartimento di Chimica, Materiali e Ingegneria Chimica "Giulio Natta", Politecnico di Milano, 20133 Milano, Italy

Simone Fabiano - Laboratory of Organic Electronics, Department of Science and Technology, Linköping University, SE-60174 Norrköping, Sweden; 이이.org/0000-00017016-6514

Mario Caironi - Center for Nano Science and Technology @ PoliMi, Istituto Italiano di Tecnologia, Milano 20133, Italy; (1) orcid.org/0000-0002-0442-4439

Complete contact information is available at: https://pubs.acs.org/10.1021/acs.macromol.0c00657

\section{Author Contributions}

The manuscript was written through contributions of all authors.

\section{Funding}

Funding by the DFG (Project SO 1213/8-1) is greatly acknowledged. F.L. thanks the FCI for a Liebig fellowship.

Notes

The authors declare no competing financial interest.

\section{ACKNOWLEDGMENTS}

We thank Maximilian Raisch for DSC measurements, Desiree Adamczak for SEC measurements, and Anders Mårtensson and Christian Müller (Chalmers University of Technology) for measuring elemental analysis. This work was performed in part at the SAXS/WAXS beamline ${ }^{43}$ at the Australian Synchrotron, part of ANSTO.

\section{REFERENCES}

(1) Mei, J.; Bao, Z. Side Chain Engineering in Solution-Processable Conjugated Polymers. Chem. Mater. 2014, 26, 604-615.

(2) Giovannitti, A.; Maria, I. P.; Hanifi, D.; Donahue, M. J.; Bryant, D.; Barth, K. J.; Makdah, B. E.; Savva, A.; Moia, D.; Zetek, M.; Barnes, P. R. F.; Reid, O. G.; Inal, S.; Rumbles, G.; Malliaras, G. G.; Nelson, J.; Rivnay, J.; McCulloch, I. The Role of the Side Chain on the Performance of N-Type Conjugated Polymers in Aqueous Electrolytes. Chem. Mater. 2018, 30, 2945-2953.

(3) Meng, B.; Song, H.; Chen, X.; Xie, Z.; Liu, J.; Wang, L. Replacing Alkyl with Oligo(Ethylene Glycol) as Side Chains of Conjugated Polymers for Close $\pi-\pi$ Stacking. Macromolecules 2015, $48,4357-4363$.

(4) Giovannitti, A.; Nielsen, C. B.; Sbircea, D.-T.; Inal, S.; Donahue, M.; Niazi, M. R.; Hanifi, D. A.; Amassian, A.; Malliaras, G. G.; Rivnay, J.; McCulloch, I. N-Type Organic Electrochemical Transistors with Stability in Water. Nat. Commun. 2016, 7, 13066.

(5) Kiefer, D.; Giovannitti, A.; Sun, H.; Biskup, T.; Hofmann, A.; Koopmans, M.; Cendra, C.; Weber, S.; Anton Koster, L. J.; Olsson, E.; Rivnay, J.; Fabiano, S.; McCulloch, I.; Müller, C. Enhanced N-Doping Efficiency of a Naphthalenediimide-Based Copolymer through Polar Side Chains for Organic Thermoelectrics. ACS Energy Lett. 2018, 3, $278-285$.

(6) Chen, X.; Zhang, Z.; Ding, Z.; Liu, J.; Wang, L. Diketopyrrolopyrrole-Based Conjugated Polymers Bearing Branched Oligo(Ethylene Glycol) Side Chains for Photovoltaic Devices. Angew. Chem., Int. Ed. 2016, 55, 10376-10380.

(7) Khan, A.; Müller, S.; Hecht, S. Practical Synthesis of an Amphiphilic, Non-Ionic Poly(Para-Phenyleneethynylene) Derivative with a Remarkable Quantum Yield in Water. Chem. Commun. 2005, 584-586.

(8) Cataldi, P.; Cassinelli, M.; Heredia-Guerrero, J. A.; GuzmanPuyol, S.; Naderizadeh, S.; Athanassiou, A.; Caironi, M. Green Biocomposites for Thermoelectric Wearable Applications. Adv. Funct. Mater. 2020, 30, 1907301. 
(9) Gaikwad, A. M.; Khan, Y.; Ostfeld, A. E.; Pandya, S.; Abraham, S.; Arias, A. C. Identifying Orthogonal Solvents for Solution Processed Organic Transistors. Org. Electron. 2016, 30, 18-29.

(10) Zakhidov, A. A.; Lee, J.-K.; DeFranco, J. A.; Fong, H. H.; Taylor, P. G.; Chatzichristidi, M.; Ober, C. K.; Malliaras, G. G. Orthogonal Processing: A New Strategy for Organic Electronics. Chem. Sci. 2011, 2, 1178.

(11) Baeg, K.-J.; Facchetti, A.; Noh, Y.-Y. Effects of Gate Dielectrics and Their Solvents on Characteristics of Solution-Processed NChannel Polymer Field-Effect Transistors. J. Mater. Chem. 2012, 22, 21138-21143.

(12) Volkov, A. V.; Sun, H.; Kroon, R.; Ruoko, T.-P.; Che, C.; Edberg, J.; Müller, C.; Fabiano, S.; Crispin, X. Asymmetric Aqueous Supercapacitor Based on P- and n-Type Conducting Polymers. ACS Appl. Energy Mater. 2019, 2, 5350-5355.

(13) Kroon, R.; Kiefer, D.; Stegerer, D.; Yu, L.; Sommer, M.; Müller, C. Polar Side Chains Enhance Processability, Electrical Conductivity, and Thermal Stability of a Molecularly p-Doped Polythiophene. Adv. Mater. 2017, 29, 1700930.

(14) Guo, X.; Watson, M. D. Conjugated Polymers from Naphthalene Bisimide. Org. Lett. 2008, 10, 5333-5336.

(15) Yan, H.; Chen, Z.; Zheng, Y.; Newman, C.; Quinn, J. R.; Dötz, F.; Kastler, M.; Facchetti, A. A High-Mobility Electron-Transporting Polymer for Printed Transistors. Nature 2009, 457, 679-686.

(16) Kim, R.; Kang, B.; Sin, D. H.; Choi, H. H.; Kwon, S.-K.; Kim, Y.-H.; Cho, K. Oligo(Ethylene Glycol)-Incorporated Hybrid Linear Alkyl Side Chains for n-Channel Polymer Semiconductors and Their Effect on the Thin-Film Crystalline Structure. Chem. Commun. 2015, 51, 1524-1527.

(17) Liu, J.; Qiu, L.; Alessandri, R.; Qiu, X.; Portale, G.; Dong, J.; Talsma, W.; Ye, G.; Sengrian, A. A.; Souza, P. C. T.; Loi, M. A.; Chiechi, R. C.; Marrink, S. J.; Hummelen, J. C.; Koster, L. J. A. Enhancing Molecular N-Type Doping of Donor-Acceptor Copolymers by Tailoring Side Chains. Adv. Mater. 2018, 30, 1704630.

(18) Liu, J.; Ye, G.; van der Zee, B.; Dong, J.; Qiu, X.; Liu, Y.; Portale, G.; Chiechi, R. C.; Koster, L. J. A. N-Type Organic Thermoelectrics of Donor-Acceptor Copolymers: Improved Power Factor by Molecular Tailoring of the Density of States. Adv. Mater. 2018, 30, 1804290 .

(19) Wang, S.; Sun, H.; Erdmann, T.; Wang, G.; Fazzi, D.; Lappan, U.; Puttisong, Y.; Chen, Z.; Berggren, M.; Crispin, X.; Kiriy, A.; Voit, B.; Marks, T. J.; Fabiano, S.; Facchetti, A. A Chemically Doped Naphthalenediimide-Bithiazole Polymer for n-Type Organic Thermoelectrics. Adv. Mater. 2018, 30, 1801898.

(20) Wang, S.; Fazzi, D.; Puttisong, Y.; Jafari, M. J.; Chen, Z.; Ederth, T.; Andreasen, J. W.; Chen, W. M.; Facchetti, A.; Fabiano, S. Effect of Backbone Regiochemistry on Conductivity, Charge Density, and Polaron Structure of n-Doped Donor-Acceptor Polymers. Chem. Mater. 2019, 31, 3395-3406.

(21) Wang, S.; Fabiano, S.; Himmelberger, S.; Puzinas, S.; Crispin, X.; Salleo, A.; Berggren, M. Experimental Evidence That Short-Range Intermolecular Aggregation Is Sufficient for Efficient Charge Transport in Conjugated Polymers. Proc. Natl. Acad. Sci. 2015, 112, 10599-10604.

(22) Rivnay, J.; Inal, S.; Salleo, A.; Owens, R. M.; Berggren, M.; Malliaras, G. G. Organic Electrochemical Transistors. Nat. Rev. Mater. 2018, 3, 1-14.

(23) Muench, S.; Wild, A.; Friebe, C.; Häupler, B.; Janoschka, T.; Schubert, U. S. Polymer-Based Organic Batteries. Chem. Rev. 2016, 116, 9438-9484.

(24) Lopez, J.; Mackanic, D. G.; Cui, Y.; Bao, Z. Designing Polymers for Advanced Battery Chemistries. Nat. Rev. Mater. 2019, 4, 312-330.

(25) O’Driscoll, L. J.; Welsh, D. J.; Bailey, S. W. D.; Visontai, D.; Frampton, H.; Bryce, M. R.; Lambert, C. J. Reversible Thermal Switching of Aqueous Dispersibility of Multiwalled Carbon Nanotubes. Chem. - Eur. J. 2015, 21, 3891-3894.

(26) Hansen, M. R.; Schnitzler, T.; Pisula, W.; Graf, R.; Müllen, K.; Spiess, H. W. Cooperative Molecular Motion within a Self-Assembled
Liquid-Crystalline Molecular Wire: The Case of a TEG-Substituted Perylenediimide Disc. Angew. Chem., Int. Ed. 2009, 48, 4621-4624.

(27) Kota, R.; Samudrala, R.; Mattern, D. L. Synthesis of Donor- $\sigma$ Perylenebisimide-Acceptor Molecules Having PEG Swallowtails and Sulfur Anchors. J. Org. Chem. 2012, 77, 9641-9651.

(28) Samudrala, R.; Zhang, X.; Wadkins, R. M.; Mattern, D. L. Synthesis of a Non-Cationic, Water-Soluble Perylenetetracarboxylic Diimide and Its Interactions with G-Quadruplex-Forming DNA. Bioorg. Med. Chem. 2007, 15, 186-193.

(29) Wyszogrodzka, M.; Haag, R. Study of Single Protein Adsorption onto Monoamino Oligoglycerol Derivatives: A Structure-Activity Relationship. Langmuir 2009, 25, 5703-5712.

(30) Matsidik, R.; Komber, H.; Luzio, A.; Caironi, M.; Sommer, M. Defect-Free Naphthalene Diimide Bithiophene Copolymers with Controlled Molar Mass and High Performance via Direct Arylation Polycondensation. J. Am. Chem. Soc. 2015, 137, 6705-6711.

(31) Goto, E.; Ando, S.; Ueda, M.; Higashihara, T. Nonstoichiometric Stille Coupling Polycondensation for Synthesizing Naphthalene-Diimide-Based $\pi$-Conjugated Polymers. ACS Macro Lett. 2015, 4, 1004-1007.

(32) Nübling, F.; Komber, H.; Sommer, M. All-Conjugated, AllCrystalline Donor-Acceptor Block Copolymers P3HT-b-PNDIT2 via Direct Arylation Polycondensation. Macromolecules 2017, 50, 1909-1918.

(33) Sanzone, A.; Cimò, S.; Mattiello, S.; Ruffo, R.; Facchinetti, I.; Bonacchini, G. E.; Caironi, M.; Sassi, M.; Sommer, M.; Beverina, L. Preparation of Naphthalene Dianhydride Bithiophene Copolymers by Direct Arylation Polycondensation and the Latent Pigment Approach. ChemPlusChem 2019, 84, 1346-1352.

(34) Steyrleuthner, R.; Schubert, M.; Howard, I.; Klaumünzer, B.; Schilling, K.; Chen, Z.; Saalfrank, P.; Laquai, F.; Facchetti, A.; Neher, D. Aggregation in a High-Mobility n-Type Low-Bandgap Copolymer with Implications on Semicrystalline Morphology. J. Am. Chem. Soc. 2012, 134, 18303-18317.

(35) Robitaille, A.; Jenekhe, S. A.; Leclerc, M. Poly(Naphthalene Diimide-Alt-Bithiophene) Prepared by Direct (Hetero)Arylation Polymerization for Efficient All-Polymer Solar Cells. Chem. Mater. 2018, 30, 5353-5361.

(36) Dhanabalan, A.; van Dongen, J. L. J.; van Duren, J. K. J.; Janssen, H. M.; van Hal, P. A.; Janssen, R. A. J. Synthesis, Characterization, and Electrooptical Properties of a New Alternating N-Dodecylpyrrole-Benzothiadiazole Copolymer. Macromolecules 2001, 34, 2495-2501.

(37) Zhang, X.; Qu, Y.; Bu, L.; Tian, H.; Zhang, J.; Wang, L.; Geng, Y.; Wang, F. Synthesis and Characterization of Monodisperse Oligo(Fluorene-Co-Bithiophene)s. Chem. - Eur. J. 2007, 13, 62386248.

(38) Brinkmann, M.; Gonthier, E.; Bogen, S.; Tremel, K.; Ludwigs, S.; Hufnagel, M.; Sommer, M. Segregated versus Mixed Interchain Stacking in Highly Oriented Films of Naphthalene Diimide Bithiophene Copolymers. ACS Nano 2012, 6, 10319-10326.

(39) Denti, I.; Cimò, S.; Brambilla, L.; Milani, A.; Bertarelli, C.; Tommasini, M.; Castiglioni, C. Polaron Confinement in N-Doped P(NDI2OD-T2) Unveiled by Vibrational Spectroscopy. Chem. Mater. 2019, 31, 6726-6739.

(40) Saglio, B.; Mura, M.; Massetti, M.; Scuratti, F.; Beretta, D.; Jiao, X.; McNeill, C. R.; Sommer, M.; Famulari, A.; Lanzani, G.; Caironi, M.; Bertarelli, C. N-Alkyl Substituted $1 \mathrm{H}$-Benzimidazoles as Improved n-Type Dopants for a Naphthalene-Diimide Based Copolymer. J. Mater. Chem. A 2018, 6, 15294-15302.

(41) Shin, Y.; Massetti, M.; Komber, H.; Biskup, T.; Nava, D.; Lanzani, G.; Caironi, M.; Sommer, M. Improving Miscibility of a Naphthalene Diimide-Bithiophene Copolymer with n-Type Dopants through the Incorporation of "Kinked" Monomers. Adv. Electron. Mater. 2018, 4, 1700581.

(42) Schlitz, R. A.; Brunetti, F. G.; Glaudell, A. M.; Miller, P. L.; Brady, M. A.; Takacs, C. J.; Hawker, C. J.; Chabinyc, M. L. SolubilityLimited Extrinsic n-Type Doping of a High Electron Mobility 
Polymer for Thermoelectric Applications. Adv. Mater. 2014, 26, $2825-2830$.

(43) Kirby, N. M.; Mudie, S. T.; Hawley, A. M.; Cookson, D. J.; Mertens, H. D. T.; Cowieson, N.; Samardzic-Boban, V. A LowBackground-Intensity Focusing Small-Angle X-Ray Scattering Undulator Beamline. J. Appl. Crystallogr. 2013, 46, 1670-1680. 